\title{
Digital Image Watermarking using 3-Level DWT and FFT via Image Compression
}

\author{
Reema Jain \\ M.Tech. Student \\ Dept. of Electronics \& Communication Engg. \\ Modi Institute of Technology, Kota, India
}

\author{
Manish Jain, PhD \\ Associate Professor and Head \\ Dept. of Electronics \& Communication Engg. \\ Modi Institute of Technology, Kota, India
}

\begin{abstract}
Today is an era of the Internet. The day is not so far when the whole data of the world will travel on the internet. So, authentication is required to protect the content of the data against unauthorized copying and replication. Information hiding via digital watermarks for the multimedia data plays a vital role in protecting the data. Watermarks are imperceptible and it is a predefined pattern inserted into the multimedia data to protect its authenticity. The watermark indicates that data is containing a copyright or not. The main focus of researchers is to develop an algorithm which has robustness against attacks and induced embedded distortions. Many digital watermarking mechanisms have been proposed in spatial and transform domain. The hybrid approaches of combining different transform domain techniques have garnered attention recently. This paper presents design of digital image watermarking using hybrid 3-level DWT-FFT technique via image compression for better results as compared to previous techniques of information hiding.
\end{abstract}

\section{General Terms}

Digital Image Watermarking, Robust Watermarking, Copyright Protection

\section{Keywords}

Digital Image Watermarking, Robust Watermarking, Discrete Wavelet Transform (DWT), Fast Fourier Transform (FFT), Fractional Fourier Transform (FRFT), Peak Signal to Noise Ratio (PSNR), Joint Photographic Expert Group (JPEG)

\section{INTRODUCTION}

Today's world is digital world. The rapid expansion of the internet in the past years has rapidly increased the availability of digital data such as image, audio and video to the public. The problem of protecting multimedia information becomes more and more subtle and a lot of research is concerned about protecting any illegal duplication of data or work. This is an interesting challenge and that is probably why so much attention has been drawn towards the development of digital information protection schemes.

There are many approaches possible to protect visual data. Digital watermarking is the one that has received more attention. In digital watermarking, a watermark is embedded into a cover image in such a way that the resulting watermarked signal is robust to certain distortions caused by either standard data processing e.g. JPEG compression in a friendly environment or malicious attacks in an unfriendly environment. Watermarking is the process of inserting predefined patterns into multimedia data in such a way that the degradation of quality is minimized and remains at an imperceptible level. The watermark indicates that data is containing a copyright or not $[1,2]$.
Information hiding and increase in the utilization of digital watermark in numerous applications such as multimedia, electronics and communication etc. [3] has enhanced the requirement of an efficient method that can accumulate and convey that information. This requirement formulates the image compression \& quantization as an essential factor and has increased the need for efficient algorithms that can result in high compression ratio with minimum losses.

Many digital watermarking techniques have been proposed in spatial and transform domains. The spatial domain techniques still have relatively low bit capacity and are not resistant enough to lossy image compression and other image processing operations $[4,5]$. A simple compression of the image may eliminate the watermark. On the other side, frequency domain based techniques can embed more bits of watermark information and so are more robust to attacks. Some transforms like Discrete Cosine Transform (DCT), Discrete Wavelet Transform (DWT) and Fast Fourier Transform (FFT) are used for watermarking in the frequency domain $[6,7]$.

In this paper, comparative implementation of frequency domain watermarking techniques are discussed in a very concise manner. From the two existing DWT and FFT techniques, one hybrid technique is developed. This work provides Digital Image Watermarking based on Hybrid DWTFFT technique via image compression. Peak Signal to Noise Ratio (PSNR) is computed to measure image quality of our proposed technique for better results as compared to previous techniques of information hiding.

Finally, the performance of the digital watermarking scheme is evaluated as tradeoffs between images embedded on it and robustness against image compression. The remainder of the paper is organized as follows: In Section II, classification of watermarking technique is described. Section III explains the basic requirement of a digital image watermarking algorithm. Next, Section IV presents the proposed method. Section V describes the detailed experimental results \& its comparison. And in the last Section, conclusion is given.

\section{CLASSIFICATION OF WATERMARK ALGORITHMS}

Based on the human perception, watermark algorithms are divided into two categories [3]:

\subsection{Visible Watermarking (alpha>0.1):}

In visible watermarking, the embedded watermark is easily percept by the human eye. It means that the watermark can be seen without the extraction process. For example, it can be name or logo of the company. 


\subsection{Invisible Watermarking (alpha<0.1):}

In this watermarking, the watermark cannot be seen by human eye. It is embedded in the data without affecting the content and can be extracted by the owner only.

\section{Robust Watermark:}

If the watermark can survive even after the common signal processing operations like filtering and lossy compression etc. it is said to be a robust watermark.

\section{BASIC REQUIREMENT FOR A DIGITAL WATERMARK ALGORITHM}

All watermarks contain some important information so watermark cannot be stored in the file header because anyone from the computer can get the digital editing and would be able to convert the basic information and can remove the watermark at the same time. Thus, the watermark should always be embedded into the multimedia signals [5].

As shown in Fig 1, original multimedia data and the watermark are the inputs to the algorithm. Watermarked data is the output of the algorithm, which consists of the secret key and the original data, see Fig 2 .

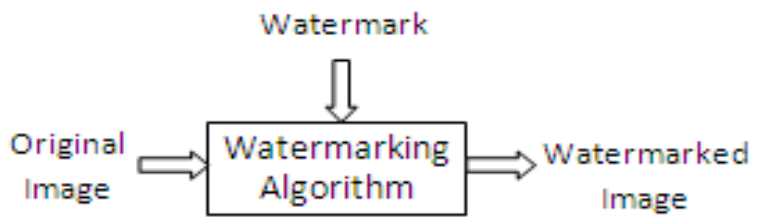

Fig 1: Watermark Embedding Process

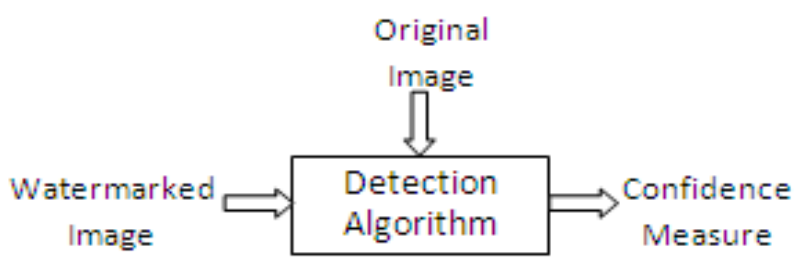

Fig 2: Watermark Extraction Process

Properties of a watermark depend on the type of application to be used. The most important requirements for digital image watermarking can be summarized as:

\subsection{Perceptual Transparency}

This refers to invisibility of the watermark. The watermark should not degrade the quality of the data. The watermark should be imperceptible. Sometimes watermark is embedded to data in the way that can be seen without extraction. These types of watermark are called the visible watermarks. Forexample - The logo of a company.

\subsection{Robustness}

This refers to the strength of the watermark against attacks. It means nobody is able to remove, alter or damage the watermark without the secret key. A Robust watermark would be detectable even after common signal processing operation such as lossy compression, spatial filtering, translations and rotation operations. An algorithm should be developed which can maintain the robustness of the watermark. If the digital watermark is still detectable, it is called the robust watermarking algorithm.

\subsection{Security}

Security refers to the watermark withstand capability against attacks like compression and noise. They are directly pointed to embed information. Secret key determines the value of watermark and the location where the watermark is embedded. It must not be possible to retrieve or even modify the watermark without knowledge of the secret key.

\subsection{Compression}

Practically all images currently being distributed via Internet have been compressed. The watermark is required to resist at different levels of compression. It is usually advisable to perform the embedding of watermark in the same domain where the compression takes place [8].

\section{PROPOSED HYBRID 3-LEVEL DWT- FFT TECHNIQUE FOR DIGITAL IMAGE WATERMARKING}

\subsection{Fractional Fourier Transform (FRFT)}

Recently Fractional Fourier Transform (FRFT) has been widely used as a powerful tool in signal processing, quantum mechanics, quantum optics, pattern recognition and study of time frequency distribution [5]. The FRFT can be interpreted as the rotation of angle $\theta$ in the time frequency plane. The basic properties of FRFT are- when rotation angle $\theta=\pi / 2$, it corresponds to the classical Fourier Transform, if $\theta=0$, it corresponds to an identity operator and when we apply FRFT on the signal, the signal decomposes into chirps i.e., complex exponentials with linearly varying instantaneous frequencies [9].

We use common method to embed watermarking signals in spatial and frequency domain. We can also combine spatial and frequency domain. This type of watermarking is considered image watermarking in the Fractional Fourier Transformation FRFT domain. Here, we use the combination of time and frequency domains [5,9].

In this way, we are able to create more watermarks than the FFT or DCT domain [10]. We use different angles for embedding watermark. This watermarking is robust against some important attacks (such as geometrical transform, filtering, histogram stretching etc.) that could be performed by a pirate. Suppose that the pirate knows watermark key and its position then also he is not able to get the transformation angle without owner's permission [5].

\subsection{Discrete Wavelet Transform (DWT)}

The wavelet transform represents a signal in terms of mother wavelets using dilation and translation $[6,11]$. The features extracted by wavelet transform gives better results in recognition as well as in bifurcating low frequency and high frequency components as approximation band and detailed bands respectively. Advantages of Discrete Wavelet Transform are: It gives information about both time and frequency of the signal, transform of a non-stationary signal is efficiently obtained, reduces the size without losing much of resolution, reduces redundancy and reduces computational time also.

\subsection{Fast Fourier Transform (FFT)}

The FFT is applied on spatial domain image to obtain FFT coefficients $[9,10]$. The features that are extracted from FFT coefficients are real part, imaginary part, magnitude value and 
phase angle. The FFT computation is fast compared to Discrete Fourier Transform (DFT) [10], since the number of multiplications required to compute N-point DFT are less i.e., only $(\mathrm{N} / 2)\left[\log _{2} \mathrm{~N}\right]$ in FFT as against $\mathrm{N}^{2}$ in DFT. The features of DWT are obtained from approximation band only. The features of FFT are computed using the magnitude values.

In our proposed technique, first we have applied Hybrid 3Level DWT-FFT technique on both original image and watermark image. Further, we applied the watermarking algorithm as shown in Fig 1. Then, inverse hybrid 3-Level DWT-FFT (as 3-Level IDWT-FFT) is applied, Then, we get a final watermarked image.

In unsecure environments, this watermarked image is vulnerable to many types of attacks and can become noisy and distorted etc. In secure environments also, during JPEG compression, it can lose its quality and become blurred. In retrieval process to extract the watermark from watermarked image, we use the detection algorithm as shown in Fig 2.

\section{EXPERIMENTAL RESULTS OF PROPOSED TECHNIQUE}

The cover image is bike.jpg as shown Fig 3 .

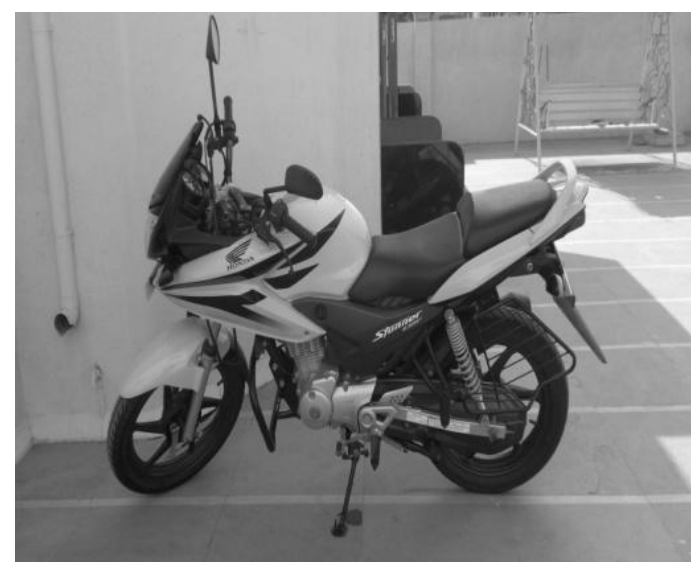

Fig 3: Cover Image

Embedding watermark image cherry.jpg is shown Fig 4.

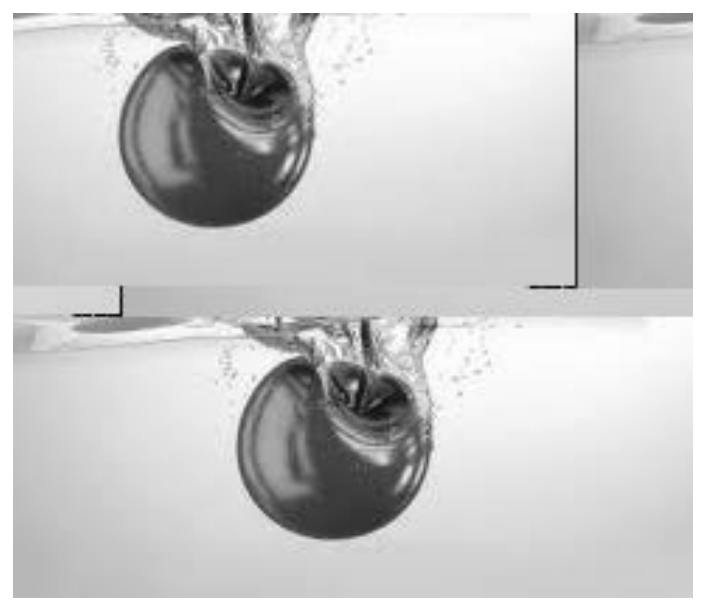

Fig 4: Watermark Image
Table 1: Varying alpha and constant $q$

\begin{tabular}{|c|c|c|c|}
\hline Alpha & $\begin{array}{c}\text { q(quality } \\
\text { factor) }\end{array}$ & $\begin{array}{c}\text { FRFT } \\
\text { (PSNR) }\end{array}$ & $\begin{array}{c}\text { FFT } \\
\text { (PSNR) }\end{array}$ \\
\hline 0 & 80 & 42.2128 & 42.2128 \\
\hline 0.01 & 80 & 37.1706 & 37.1682 \\
\hline 0.03 & 80 & 29.2895 & 29.2874 \\
\hline 0.05 & 80 & 25.1355 & 25.1265 \\
\hline 0.1 & 80 & 19.5993 & 19.5879 \\
\hline 0.2 & 80 & 14.3768 & 14.3768 \\
\hline
\end{tabular}

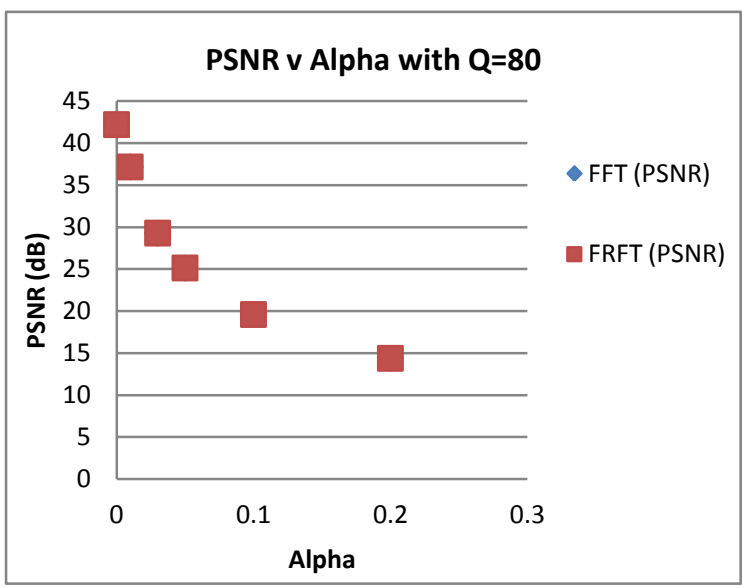

Fig 5: Chart for PSNR v/s alpha with constant q

Table 2: Constant alpha and varying $q$

\begin{tabular}{|c|c|c|}
\hline Alpha & 0.0001 & 0.0001 \\
\hline q(quality factor) & 90 & 100 \\
\hline FRFT PSNR & 25.1732 & 25.1674 \\
\hline FFT PSNR & 25.2007 & 25.1954 \\
\hline MSE & $7.9674 \mathrm{e}-009$ & 50.9813 \\
\hline DWT PSNR & 186.5979 & 2.6480 \\
\hline
\end{tabular}

PSNR v Quality (q) with alpha $=0.05$

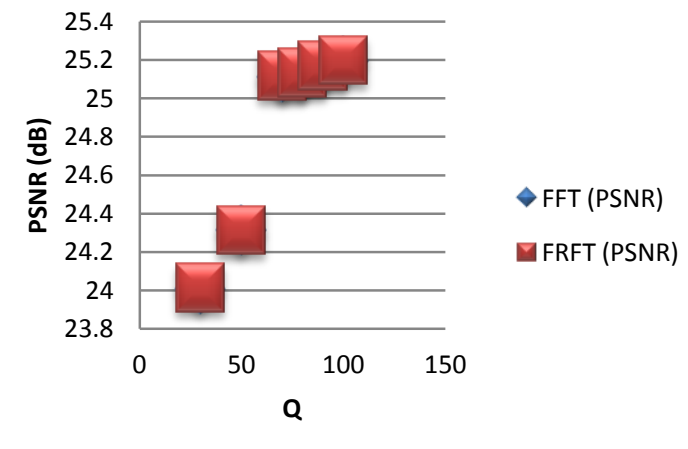

Fig 6: Chart for PSNR v/s q with constant alpha 


\section{CONCLUSION}

In this paper, we discussed watermarking technique using hybrid 3-level DWT-FFT technique via image compression. We notice that the process is the same but we apply different transform technique. We can note that FRFT is more robust than FFT due to PSNR of FRFT based watermarking technique. But after comparing it with DWT PSNR, the results of DWT are much better as compared to FFT \& FRFT based watermarking technique.

In future, we can apply other frequency domain techniques in hybrid manner like DCT based Laplacian transform etc. and can improve the PSNR value \& reduce MSE even further.

\section{REFERENCES}

[1] Edin Muharemagic and Borko Furht, "Survey of Watermarking Techniques and Applications", Department of Computer Science and Engineering, Florida Atlantic University.

[2] Andreja Sam̌covi'c, J'an Tur'an, "Attacks on Digital Wavelet Image Watermarks", Journal of Electrical Engineering.

[3] Peining Taoa and Ahmet M. Eskicioglub, "A robust multiple watermarking scheme in the Discrete Wavelet Transform Domain", The Graduate Center, The City University of New York.

[4] Baisa L. Gunjal, "An Overview of Transform Domain Robust Digital Image Watermarking Algorithms", Department of Computer Engineering, Amrutvahini College of Engineering.
[5] Igor Djurovic, Srdjan Stankovic and Ioannis Pitas, "Digital watermarking in the Fractional Fourier Transformation domain", Journal of Network and Computer Applications, 2001, page 167-173.

[6] Vaishali S. Jabade, Dr. Sachin R. Gengaje, "Literature Review of Wavelet Based Digital Image Watermarking Techniques", International Journal of Computer Applications, Vol. 31, No.1, October-2011.

[7] P. Meerwald, A. Uhl, "A Survey of Wavelet-Domain Watermarking Algorithms", EI San Jose, CA, USA, 2001.

[8] Mohamed A. Suhail and Mohammad S. Obaidat, "Digital Watermarking-Based DCT and JPEG Model", IEEE Transactions On Instrumentation and Measurement, Vol. 52, Issue 5, pp.1640-1647, October 2003.

[9] Mahendra Kumar et.al., "Digital Image Watermarking using Fractional Fourier Transform via Image Compression", In IEEE International Conference on Computational Intelligence and Computing Research ICCIC 2013, 26-28 Dec. 2013.

[10] Mahendra Kumar et al., "Digital image watermarking: A survey", International Journal of Engineering and Research Applications IJERA, Jul-Aug 2013.

[11] John G. Proakis, Dimitris K. Manolakis, "Digital Signal Processing", 4th Edition, ISBN-13: 978-0131873742, 2006. 